\title{
Is disomic homozygosity at the APECED locus the cause of increased autoimmunity in Down's syndrome?
}

\author{
Julian P H Shield, Emma J K Wadsworth, Terry J Hassold, Lu Ann Judis, Patricia A Jacobs
}

Institute of Child

Health, University of Bristol, St Michael's Hill, Bristol BS2 8BJ, UK

J P H Shield

E J K Wadsworth

Department of Genetics and the Centre for Human

Genetics, Case

Western Reserve

University School of

Medicine, and the

University Hospital, Cleveland, OH 44106, USA

T J Hassold

L A Judis

Wessex Regional

Genetics Laboratory, Salisbury District

Hospital, Salisbury SP2 8BJ, UK

P A Jacobs

Correspondence to: Dr Shield. email: J.P.H.Shield@

bris.ac.uk

Accepted 3 March 1999

\begin{abstract}
Aims-To examine the age of onset of insulin dependent diabetes mellitus (IDDM) in children with Down's syndrome compared with non-trisomic individuals, and to assess whether differences might be related to disomic homozygosity at the autoimmune polyglandular disease type 1 (APECED) gene locus. Methods-Children with Down's syndrome and IDDM were identified through the Down's syndrome association newsletter and from paediatricians. DNA was extracted from mouthbrush preparations provided by the parents and patients using standard techniques. Mapping techniques were then used to identify areas of reduction to homozygosity, including a marker that overlaps the locus for APECED. The frequency of disomic homozygosity for all markers $(n=18)$ was compared with a control group of 99 patients with Down's syndrome and their parents. The families also answered a questionnaire concerning diabetes and related autoimmune conditions in the family. Details were compared with the British Paediatric Surveillance Group 1988 diabetes study.

Results-Children with Down's syndrome and IDDM were diagnosed significantly earlier than the general population (6.7 $v$ 8.0 years) with a far higher proportion diagnosed in the first 2 years of life $(22 \% v$ $7 \%$ ). There was no evidence of increased disomic homozygosity in the region of the APECED locus in Down's syndrome patients with IDDM compared with simple Down's syndrome.

Conclusions-The natural history of IDDM in Down's syndrome is different from that of the general population. Although children with Down's syndrome have features similar to cases of APECED, disomic homozygosity in this region does not explain the predilection for autoimmune disease. (Arch Dis Child 1999;81:147-150)
\end{abstract}

Keywords: autoimmune polyglandular disease type 1 locus; Down's syndrome; insulin dependent diabetes mellitus

It is well established that there is an increased prevalence of insulin dependent diabetes mellitus (IDDM) in Down's syndrome. ${ }^{1-3}$ Furthermore, a study in the 1960 s suggested an earlier peak age of onset in children with Down's syn- drome compared with non-trisomic children (8 years $v 14$ years). ${ }^{4}$ The increased prevalence might be associated with trisomy 21 but, to date, no susceptibility loci have mapped to this chromosome, ${ }^{5}$ so a permissive gene has yet to be found to explain this increased risk. In addition to an increased incidence of IDDM, Down's syndrome also confers an increased risk for a number of other autoimmune related phenomena, namely thyroid disease, alopecia, vitiligo, and chronic active hepatitis, ${ }^{6}$ and has been described with hypoparathyroidism. ${ }^{7}$ Oral candidiasis with clinically apparent erythematous or pseudomembranous lesions is far more prevalent in children with Down's syndrome $(40 \%)$ compared with normal controls $(<1 \%),{ }^{8}$ as is nail and dental enamel dystrophy.

A rare autosomal recessive condition, autoimmune polyglandular syndrome type 1 (APECED) has been mapped recently to chromosome $21 \mathrm{q} 22.3$. In this condition, patients have an increased susceptibility to autoimmune endocrine disease of the parathyroid, thyroid, pancreas, and adrenal cortex. Other features include mucocutaneous candidiasis, alopecia, hepatitis, and dental and nail dystrophy. ${ }^{9}$ Mutations in a novel gene AIRE have now been identified within this region causing APECED. ${ }^{10}$

Feingold et al have suggested recently that some abnormalities of Down's syndrome are attributable to overexpression at specific loci, resulting from the presence of two identical copies of a susceptibility allele inherited from the parent of origin of trisomy (disomic homozygosity). By inheriting two identical copies of a rare susceptibility allele the suggestion is that an individual with Down's syndrome exceeds a liability threshold and manifests the disorder. ${ }^{11}$

Our study was designed to examine any change since the age of onset of IDDM in children with Down's syndrome was reported in 1969 , given the apparent major shift in age of onset for the general population over the past 20 years. ${ }^{12}$ We examined the possibility that the increased manifestation of autoimmune related phenomena, which in many respects are similar to those seen in APECED, might be related to disomic homozygosity in the region $21 \mathrm{q} 22.3$.

Methods and materials

We identified children with Down's syndrome and IDDM diagnosed before the age of 25 years from two sources. An initial request for recruits was published in the Down's syndrome 
association newsletter. Parents contacted the researchers directly and were asked to complete a questionnaire regarding the clinical details of the patient and direct family. They were sent kits with which to take buccal smears for DNA extraction to analyse disomic homozygosity. To obtain more cases for epidemiological analysis, all consultants who participated in both the 1988 and 1992 British Paediatric Surveillance Unit (BPSU) surveys of diabetes ${ }^{13}{ }^{14}$ were asked if they were willing to identify children under their care with IDDM and Down's syndrome. Depending on their response, parents were then provided with a questionnaire. Approval was obtained from the local ethics committee.

MOLECULAR STUDIES

DNA from patients with Down's syndrome and their parents was obtained from mouthbrush preparations, using standard extraction techniques. We conducted molecular analyses of the parent and timing of origin of nondysjunction using previously described techniques, ${ }^{15}$ analysing 18 polymerase chain reaction (PCR) based chromosome 21 polymorphisms (cen to tel: D21S369, D21S215, D21S258， D21S120，D21S192，D21S11, D21S214，D21S232，D21S210，D21S226, D21S167, D21S156, D21S416, D21S1224, D21S212, D21S1225, PFKL, and D21S171); we obtained primer sequences from the genome database.

We inferred the meiotic stage of origin of non-dysjunction for all individuals with Down's syndrome using the pericentromeric markers D21S369, D21S215, D21S258, D21S120, or D21S192. We first studied the parent of origin of trisomy to determine at which, if any, of the markers he or she was heterozygous. If the proband inherited both alleles (non-reduction or "N") at one or more of these markers we concluded a meiosis I error, if the proband inherited two copies of the same allele (reduction to homozygosity or "R") at one or more markers, we concluded a meiosis II or mitotic error. If the proband had both reduced and non-reduced loci in the proximal region, the one nearest the centromere was used to infer the stage of origin.

To distinguish between meiosis II and mitotic errors, we examined other, nonproximal loci. If the proband was non-reduced at any of these, we concluded a meiosis II error had taken place. However, if the proband was reduced at all informative markers, including at least one each in proximal, medial, and distal 21q, we concluded a mitotic error had occurred.

We used centromere mapping techniques to determine the degree of recombination between the non-dysjoined chromosomes 21 , as well as the relative frequency of reduced and non-reduced loci. ${ }^{16}$ In brief, the approach involves analyses of pairs of chromosome 21 markers to determine whether both markers are non-reduced $(\mid \mathrm{N}>\mathrm{N})$, both are reduced $(\mathrm{R}>\mathrm{R})$, or one is non-reduced and the other reduced $(\mathrm{N}>\mathrm{R}$ or $\mathrm{R}>\mathrm{N})$. The first two situations are consistent with no recombination between the markers, whereas the third indicates that recombination has occurred. By analysing ordered pairs of markers from proximal to distal $21 \mathrm{q}$, it is possible to determine the minimal number, as well as the location, of exchanges between the non-dysjoined chromosomes $21 .^{17}$

For individuals with Down's syndrome and IDDM, we calculated the frequency of disomic homozygosity at each chromosome 21 marker or marker set (that is, at each marker, the number of individuals with reduced loci $\div$ number of individuals with reduced loci + number of individuals with non-reduced loci). We then compared the frequencies with those of a control Down's syndrome group, consisting of 99 individuals with Down's syndrome and their parents. The controls had been ascertained for studies of nondysjunction unrelated to the present analysis and consisted of 43 individuals with Down's syndrome and a congenital heart defect and 56 individuals with Down's syndrome but without the heart defect. The control individuals with Down's syndrome were not examined for IDDM; nevertheless, only a small fraction would be expected to be IDDM positive.

\section{Results}

Twenty one families were identified from the newsletter, of whom 18 returned questionnaires and 16 also returned buccal smear collections for DNA analysis. A further 60 families were identified by paediatricians and 41 families returned completed questionnaires. Overall, 59 families completed questionnaires.

Those who developed IDDM did so at a median age of 9 years. The age of onset of children under 15 who developed IDDM was compared with the 1988 BPSU national study of diabetes in children under 15 years ( $n=1600$ cases). Children with Down's syndrome were diagnosed with IDDM significantly earlier than the general population (mean age, $6.7 v 8.0$ years; $\mathrm{p}=0.02$ ). The distribution of age at diagnosis was also different between the two datasets (KolmogorovSmirnov, $\mathrm{p}=0.04)$. A significantly higher pro-

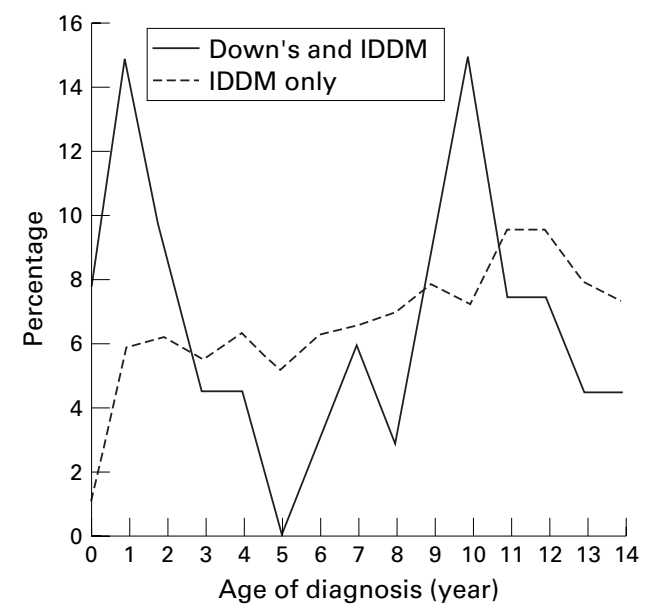

Figure 1 Variations in age of onset of insulin dependent diabetes mellitus (IDDM) between children with Down's syndrome and the 1988 British Paediatric Surveillance Unit survey. 
Table 1 Summary of results of parent and meiotic/mitotic stage of origin studies in 16 individuals with Down's syndrome and insulin dependent diabetes mellitus (IDDM) and 99 individuals with Down's syndrome not known to have IDDM

\begin{tabular}{lcc}
\hline Origin of trisomy & IDDM & Controls \\
\hline Maternal & 8 & 58 \\
Meiosis I & 3 & 22 \\
Meiosis II & 2 & 4 \\
Meiosis I or II & - & 3 \\
Paternal & 1 & 4 \\
Meiosis I & - & - \\
Meiosis II & 2 & 8 \\
Meiosis I or II & 16 & 99 \\
Mitotic & & \\
Total & & \\
\hline
\end{tabular}

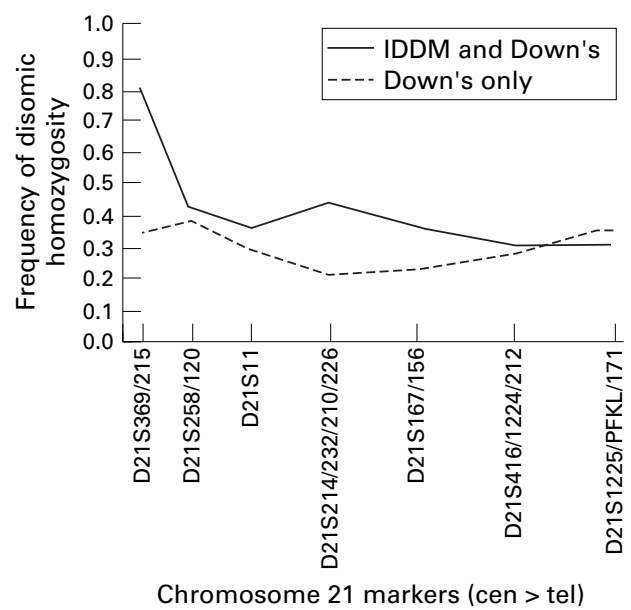

Figure 2 Comparison of the level of disomic homozygosity in individuals with Down's syndrome and insulin dependent diabetes mellitus and individuals with Down's syndrome not known to have IDDM at seven marker sets spanning the long arm of chromosome 21. Note that exon 14 of AIRE, the gene associated with autoimmune polyglandular disease type 1 (APECED), overlaps with the promoter region of PFKL (phosphofructokinase of liver type) in $21 q 22.3$.

portion of children with Down's syndrome who developed IDDM did so before their 2nd birthday (22\% v 7\%; p $<0.00001)$ (fig 1).

Seven patients had a first degree relative with IDDM (two fathers, five siblings); similar to the 1988 study. Twenty one patients with IDDM had thyroid disease, 14 had tooth or nail problems, four coeliac disease, six alopecia, and four vitiligo.

PARENT AND MEIOTIC/MITOTIC STAGE OF ORIGIN OF TRISOMY

Table 1 summarises the results of the DNA marker studies of the parent and meiotic stage of origin of trisomy. In the IDDM group, maternal non-dysjunction was the cause of the extra chromosome 21 in 13 of 16 cases, with maternal meiosis I errors accounting for the largest number of cases. In two trisomies, the additional chromosome was thought to have arisen mitotically, because all markers were reduced to homozygosity.

We found a similar distribution of nondysjunctional errors among the 99 controls. Of these, 84 were maternally derived, with most originating at maternal meiosis I. Eight of the trisomies were scored as arising in mitosis.
ANALYSIS OF DISOMIC HOMOZYGOSITY

We plotted the frequency of disomic homozygosity at the different chromosome 21 markers to determine whether or not any regions of significant variation existed between the IDDM and control Down's syndrome populations (fig 2). A tendency towards increased disomic homozygosity in the IDDM group was found in the pericentromeric region, approaching significance at marker D21S369/215.

However, we did not identify similar increases in other chromosome 21 regions and, in particular, none was evident in $21 \mathrm{q} 22.3$, the region housing APECED.

\section{Discussion}

An explanation is needed for the downward shift from the 1960s and difference in ages of onset of IDDM between children with and without Down's syndrome. It is well recognised that immune dysregulation occurs in Down's syndrome, mainly in cell mediated immunity. ${ }^{18}$ The very early peak age of onset of IDDM in Down's syndrome in our study could suggest a very aggressive autoimmune phenomenon. Alternatively, it might represent an islet cell population more susceptible to cell mediated destruction. Some support for the former hypothesis is provided by the well acknowledged propensity for other autoimmune conditions in Down's syndrome. The high prevalence of thyroid disorders in children with Down's syndrome and IDDM (one third in our study) is in keeping with the observation of Griffin $e t a l,{ }^{19}$ who highlighted a much higher prevalence rate in patients with Down's syndrome and IDDM (83\%) than that usually described in simple Down's syndrome of between $16 \%$ and $28 \%{ }^{20}$ This suggests that a subgroup of trisomic patients carry an even higher risk of aggressive autoimmune disease.

Given the similarities between many features of the APECED syndrome and those encountered in Down's syndrome, disomic homozygosity in the APECED region could have explained the predilection for autoimmunity. Although this has now been disproved, we are left with the possibility that by understanding how trisomy chromosome 21 confers an increased susceptibility to autoimmunity, we might better understand the genetics of autoimmunity in general.

This work was supported by NIH grant P01 HD32111 and the "Friends of David Ashmead".

1 Milunsky A, Neurath PW. Diabetes mellitus in Down's syndrome. Arch Environ Health 1968;17:372-6.

2 Jeremiah DE, Leyson GE, Rose T, Francis HWS, Elliot RW. Down's syndrome and diabetes. Psychol Med 1973;3:4557.

3 Van Goor JC, Massa GG, Hirasing R. Increased incidence and prevalence of diabetes mellitus in Down's syndrome [letter]. Arch Dis Child 1997;77:186.

4 Burch PRJ, Milunsky A. Early onset diabetes mellitus in the general and Down's syndrome populations. Lancet 1969;i: 554-8.

5 Gough SCL. Genetics of insulin-dependent diabetes mellitus. In: Shield JPH, Baum JD, eds. Baillieres clinical paediatrics - childhood diabetes. London: Bailliere Tindall, 1996;4:593-608.

6 Ugazio AG, Maccario R, Notarangelo LD, et al. Immunology of Down syndrome: a review. Am $\mathcal{F}$ Med Genet Suppl gy of Down sync

7 Blumberg D, AvRuskin T. Down's syndrome, autoimmune hyperthyroidism and hypoparathyroidism: a unique triad. Am F Dis Child 1987;141:1149. 
8 Carlstedt K, Krekmanova L, Dahllof G, Ericsson B, Braathen G, Modeer T. Oral carriage of candida species in diatr Dent 1996;6:95-100.

9 Aaltonen J, Bjorses P, Sandkuijl L, Perheentupa J, Peltonen L. An autosomal locus causing autoimmune disease: autoimmune polyglandular disease type 1 assigned to chromosome 21. Nat Genet 1994;8:83-7.

10 The Finnish-German APECED consortium. An autoimmune disease, APECED, caused by mutations in a nove gene featuring two PHD-type zinc-finger domains. Nat Genet 1997;17:399-403.

11 Feingold E, Lamb NE, Sherman SL. Methods for genetic linkage analysis using trisomies. Am f Hum Genet 1990;56: 475-83.

12 Gardner SG, Bingley PJ, Sawtell PA, Weeks S, Gale EAM. Rising incidence of insulin dependent diabetes in children under 5 years in the Oxford region: time trend analysis. under 5 years in the

13 Metcalfe MA, Baum JD. Incidence of insulin dependent diabetes in children aged under 15 years in the British Isles during 1988. BMF 1991;302:443-7.
14 Wadsworth EJK, Shield JPH, Hunt LP, Baum JD. Insulin dependent diabetes in the under fives: incidence and ascerI7 1995:310:700-3.

15 Shen JJ, Williams BJ, Zipursky A, et al. Cytogenetic and molecular studies of Down syndrome individuals with leukemia. Am f Hum Genet 1995;56:915-25.

16 Chakravarti A, Majumder PP, Slaugenhaupt SA, et al. Gene-centromere mapping and the study of nondisjunction in autosomal trisomies and ovarian teratomas. In: Hassold T, Epstein CJ, eds. Molecular and cytogenetic studies of nondisjunction. New York: AR Liss Inc, 1989:45-79.

17 Sherman SL, Takaesu N, Freeman SB, et al. Association between reduced recombination and nondisjunction. $A m \mathcal{F}$ Hum Genet 1991;49:608-20.

18 Ugazio AG, Maccario R, Notarangelo LD, Burgio GR. Immunology of Down syndrome: a review. Am f Med Genet Suppl 1990;7:204-12.

19 Griffin ME, Fulcher T, Nikookam K, Crowley J, Firth RG, O'Meara NM. Down's syndrome, IDDM, and hypothyroidism [letter]. Diabetes Care 1997;20:1202-3.

20 Kennedy RL, Jones TM, Cuckle HS. Down's syndrome and the thyroid. Clin Endocrinol 1992;37:471-6.

\section{Neonatal hyperinsulinism}

Neonatal hyperinsulinism is caused by either diffuse $\beta$ cell hyperplasia (nesidioblastosis) or focal adenomatous islet cell hyperplasia. The former condition has several known causes such as mutation of the sulphonylurea receptor gene or of the potassium channel gene $\left(\mathrm{K}_{\mathrm{IR}}\right.$ 6.2 ), both recessively inherited, or of the glucokinase and other genes (dominant). A mutation of the glutamate dehydrogenase gene causes both hyperinsulinism and hyperammonaemia. Focal adenomatous islet cell hyperplasia is an example of genetic imprinting and is associated with loss of the maternal copy of the sulphonylurea receptor type 1 gene on chromosome 11 (11p 15). Workers at the Hôpital des Enfants Malades in Paris (Pascale de Lonlay-Debeney and colleagues. New England fournal of Medicine 1999;340:1169-75) have described their management of 52 neonates and their ability to distinguish between the two types of hyperinsulinism and, as a consequence, to perform restricted surgery when appropriate.

Thirty babies proved at operation to have diffuse hyperplasia and 22 to have focal adenomatous hyperplasia. The clinical features in the two groups were similar, glucose infusion rates needed to maintain plasma glucose of $3.0 \mathrm{mmol} / 1$ or more were around $16 \mathrm{mg} / \mathrm{kg} / \mathrm{min}$, and surgery was performed at around 3 months. Diazoxide therapy produced little benefit.

Preoperative assessment included transhepatic catheterisation of the portal vein and selective catheterisation of the pancreatic vein with removal of blood from selective sites within the pancreas for measurement of plasma glucose, insulin, and C peptide. This was done in 26 of the 30 in the diffuse group and 19 of the 22 in the focal group. As a result, in the diffuse hyperplasia group, 17 infants were shown to have insulin hypersecretion from all areas of the pancreas, localised hypersecretion was suspected in seven, and the results were inconclusive in two. In the focal adenomatous hyperplasia group, localised hypersecretion was detected in 17 of the 19 tested, and the localisation in each case was confirmed by immediate histology of biopsy specimens at operation. The two infants in this group with inconclusive preoperative studies both had lesions confined to the body of the pancreas. The location of the lesions in the 22 infants was head $(n=9)$, isthmus $(n=3)$, body $(n=8)$, and tail $(n=2)$.

All 30 infants with diffuse hyperplasia underwent near total pancreatectomy, whereas this operation was performed on only three in the focal adenomatous hyperplasia group (the three who did not have preoperative catheter studies), the rest had partial pancreatectomy. After near total pancreatectomy for diffuse disease, 13 children had persisting hypoglycaemia, eight developed insulin dependent diabetes, and seven had abnormal glucose tolerance. After partial pancreatectomy for focal disease there was no hypoglycaemia and glucose metabolism was normal.

Preoperative studies of localised pancreatic insulin secretion together with intraoperative histology can allow identification of patients suitable for partial pancreatectomy with much improved results. 